\title{
THE ALGEBRAIC DENSITY PROPERTY FOR AFFINE TORIC VARIETIES
}

\author{
FRANK KUTZSCHEBAUCH, MATTHIAS LEUENBERGER, AND ALVARO LIENDO
}

\begin{abstract}
In this paper we generalize the algebraic density property to not necessarily smooth affine varieties relative to some closed subvariety containing the singular locus. This property implies the remarkable approximation results for holomorphic automorphisms of the AndersénLempert theory. We show that an affine toric variety $X$ satisfies this algebraic density property relative to a closed T-invariant subvariety $Y$ if and only if $X \backslash Y \neq \mathrm{T}$. For toric surfaces we are able to classify those which posses a strong version of the algebraic density property (relative to the singular locus). The main ingredient in this classification is our proof of an equivariant version of Brunella's famous classification of complete algebraic vector fields in the affine plane.
\end{abstract}

\section{INTRODUCTION}

A remarkable property of the Euclidean space of dimension at least two, that to a great extent compensates for the lack of partition of unity for holomorphic automorphisms, was discovered by Andersén and Lempert in early 1990's [And90, AL92, see also the work by Forstnerič and Rosay [FR93. Since then, the theory of Stein manifolds with very large holomorphic automorphism group is called Andersén-Lempert theory.

The property was formalized by Varolin who named it the density property (DP). A Stein manifold $X$ has the DP if the Lie algebra generated by complete holomorphic vector fields is dense (in the compact-open topology) in the space of all holomorphic vector fields on $X$. Recall that a vector field is called complete if its flow exits for all complex time and all initial conditions.

The DP allows to construct (global) automorphisms of $X$ with prescribed local properties. More precisely, any local phase flow on a Runge domain in $X$ can be approximated by (global) automorphisms. This has remarkable applications for geometric questions in complex analysis, we refer the reader to survey articles [Ros99, KK11, Kut14] and the recent book [For11]. For smooth affine algebraic varieties, the algebraic density property (ADP) was also introduced by Varolin. The ADP implies the DP, therefore it is commonly used as a tool to prove the DP.

In this paper we generalize the ADP to not necessarily smooth affine varieties relative to some closed subvariety containing the singular locus as follows: Let $X$ be an affine algebraic variety and let $X^{\text {sing }}$ be the singular locus. We also let $Y \subseteq X$ be an algebraic subvariety of $X$ containing $X^{\text {sing }}$ and let $I=I(Y) \subseteq \mathbb{C}[X]$ be the ideal of $Y$. Let $\operatorname{VF}_{\text {alg }}(X, Y)$ be the $\mathbb{C}[X]$-module of vector fields vanishing in $Y$, i.e., $\operatorname{VF}_{\text {alg }}(X, Y)=\{\partial \mid \partial(\mathbb{C}[X]) \subseteq I\}$. Let $\operatorname{Lie}_{\text {alg }}(X, Y)$ be the Lie algebra generated by all the complete vector fields in $\operatorname{VF}_{\text {alg }}(X, Y)$.

Definition 1.1. We say that $X$ has the strong ADP relative to $Y$ if $\operatorname{VF}_{\text {alg }}(X, Y)=\operatorname{Lie}_{\text {alg }}(X, Y)$. Furthermore, we say that $X$ has the ADP relative to $Y$ if there exists $\ell \geq 0$ such that $I^{\ell} \operatorname{VF}_{\text {alg }}(X, Y) \subseteq \operatorname{Lie}_{\text {alg }}(X, Y)$. With this definition, the ADP relative to $Y$ with $\ell=0$ is just the strong ADP relative to $Y$. If we let $Y=X^{\text {sing }}$ we simply say that $X$ has the strong $\mathrm{ADP}$ or the ADP, respectively.

Date: November 12, 2014.

2000 Mathematics Subject Classification: 32M05; 32M25; 14M25.

Key words: Density property, affine toric varieties, locally nilpotent derivations, holomorphic automorphisms, Lie algebras.

The first and second authors were partially supported by Schweizerischer Nationalfond Grant 200021-140235/1 and the third author was supported by Fondecyt project 11121151. 
Except for the fact that we consider not necessarily smooth varieties, the strong ADP is a version of Varolin's Definition 3.1 in Var01 of DP for the Lie subalgebra of vector fields vanishing on $Y$. Whereas for $\ell>0$ our property is slightly weaker than Varolin's definition since we generate the Lie subalgebra of vector fields vanishing on $Y$ of order at least $\ell$ using complete vector fields vanishing on $Y$ of possibly lower order than $\ell$. Still this version of the ADP has the same remarkable consequences as in Varolin version of ADP for the group of holomorphic automorphisms of $X$ fixing $Y$ pointwise (see Theorem 6.3).

In this paper we investigate the ADP for toric varieties. Our first main result is the following theorem (see Theorem 3.77).

Theorem. Let $X$ be an affine toric variety of dimension at least two and let $Y$ be a T-invariant closed subvariety of $X$ containing $X^{\text {sing }}$. Then $X$ has the ADP relative to $Y$ if and only if $X \backslash Y \neq \mathrm{T}$.

Recall that every smooth affine toric variety is isomorphic $\mathbb{C}^{k} \times\left(\mathbb{C}^{*}\right)^{n-k}$. A special case of our theorem where $X=\mathbb{C}^{n}$ and $Y$ is the union of up to $n-1$ coordinate hyperplanes has been already proven by Varolin $\operatorname{Var01}$.

It is well known that every affine toric surface different from $\mathbb{C}^{*} \times \mathbb{C}$ or $\mathbb{C}^{*} \times \mathbb{C}^{*}$ is obtained as a quotient of $\mathbb{C}^{2}$ by the action of a cyclic group. Let $d>e$ be relatively prime positive integers. We denote by $V_{d, e}$ the toric surface obtained as the quotient of $\mathbb{C}^{2}$ by the $\mathbb{Z}_{d^{-}}$action $\zeta \cdot(u, v)=\left(\zeta u, \zeta^{e} v\right)$, where $\zeta$ is a primitive $d$-th root of unity. The following theorem is our second main result (see Corollary 5.5).

Theorem. $V_{d, e}$ has the strong ADP if and only if $e$ divides $d+1$ and $e^{2} \neq d+1$.

Furthermore, for every affine toric surface our methods allow to determine the values of $\ell$ from Definition 1.1 for which $I^{\ell} \mathrm{VF}_{\text {alg }}\left(X, X^{\text {sing }}\right) \subseteq \operatorname{Lie}_{\text {alg }}\left(X, X^{\text {sing }}\right)$. The main ingredient in the proof of this theorem is an equivariant version of Brunella's famous classification of complete algebraic vector fields in the affine plane (see [Bru04]) or, equivalently, classification of complete algebraic vector fields on affine toric surfaces (see Theorem 4.10). This result might be of independent interest.

\section{VeCTOR FIELDS AND THE ALGEBRAIC DENSITY PROPERTY}

In this section we prove a general method for establishing the ADP that we later will use to show the ADP for toric varieties.

Definition 2.1. Let $X$ be an affine algebraic variety and $Y$ be a subvariety containing $X^{\operatorname{sing}}$.

(i) Let $\operatorname{Aut}(X, Y)$ be the subgroup of automorphism of $X$ stabilizing $Y$. We say that $X$ is homogeneous with respect to $Y$ if $\operatorname{Aut}(X, Y)$ acts transitively on $X \backslash Y$.

(ii) We also let $x_{0} \in X^{\mathrm{reg}}$. A finite subset $M$ of the tangent space $T_{x_{0}} X$ is called a generating set if the image of $M$ under the action of the isotropy group of $x_{0}$ in $\operatorname{Aut}(X, Y)$ generate the whole tangent space $T_{x_{0}} X$.

The following is our main tool to establish the ADP for toric varieties. It is a generalization of [KK08, Theorem 1].

Theorem 2.2. Let $X$ be an algebraic variety homogeneous with respect to some subvariety $Y \supseteq X^{\text {sing }}$. Let also $L$ be a finitely generated submodule of the $\mathbb{C}[X]$-module $\operatorname{VF}_{\text {alg }}(X, Y)$ of vector fields vanishing on $Y$. Assume that $L \subseteq \operatorname{Lie}_{\text {alg }}(X, Y)$. If the fiber of $L$ over some $x_{0} \in X \backslash Y$ contains a generating set, then $X$ has the ADP relative to $Y$.

Proof. Let $\left\{\partial_{i}\right\}$ be a finite set of vector fields in $L$ such that $\left\{\partial_{i}\left[x_{0}\right]\right\}$ is a generating set. Let now $\left\{\beta_{j}\right\} \subseteq \operatorname{Aut}(X, Y)$ be a finite collection of automorphisms fixing $x_{0}$ such that $\left\{\beta_{j}^{*}\left(\partial_{i}\right)\left[x_{0}\right]\right\}$ span the tangent space at $x_{0}$. Since change of coordinates does not change completeness of a vector field, for $\beta \in \operatorname{Aut}(X, Y)$, the finitely generated module $L_{\beta}=\beta^{*}(L)$ is again contained in 
$\operatorname{Lie}_{\text {alg }}(X, Y)$. By replacing $L$ with $\bigoplus_{j} L_{\beta_{j}}$, we can assume that $\left\{\partial_{i}\left[x_{0}\right]\right\}$ span the tangent space at $x_{0}$.

We let $A_{1}=\left\{x \in X \backslash Y \mid \operatorname{span}\left(\partial_{i}[x]\right) \neq T_{x} X\right\}$. We also let $A_{1}=\bigcup A_{1}^{j}$ be the decomposition of $A_{1}$ in irreducible components and we pick $x_{j} \in A_{1}^{j}$. Since $X$ is homogeneous with respect to $Y$, we can choose $\alpha_{j} \in \operatorname{Aut}(X, Y)$ sending $x_{0}$ to $x_{j}$. We also put $\alpha_{0}=$ Id. Let now

$$
A_{2}=\left\{x \in X \backslash Y \mid \operatorname{span}\left\{\alpha_{j}^{*}\left(\partial_{i}\right)[x] \mid \forall i, j\right\} \neq T_{x} X\right\} .
$$

By construction $\operatorname{dim} A_{1}>\operatorname{dim} A_{2}$ and so we can proceed by induction on dimension to obtain a finite collection of automorphisms $\alpha_{j} \in \operatorname{Aut}(X, Y)$ such that the collection $\left\{\alpha_{j}^{*}\left(\partial_{i}\right)[x]\right\}$ span the tangent space at every point $x \in X \backslash Y$.

We let $E=\bigoplus_{j} L_{\alpha_{j}}$. With the same argument as before, $E$ is a finitely generated $\mathbb{C}[X]$ submodule of $\operatorname{VF}_{\text {alg }}(X, Y)$ contained in $\operatorname{Lie}_{\text {alg }}(X, Y)$. By construction, we have that the fiber of $\widetilde{E}:=\mathrm{VF}_{\text {alg }}(X, Y) / E$ at every $x \in X \backslash Y$ is trivial. Hence, the support of $\widetilde{E}$ is contained in $Y$.

We define

$$
J=\operatorname{Ann}_{\mathbb{C}[X]} \widetilde{E}:=\{f \in \mathbb{C}[X] \mid f a=0 \text { for all } a \in \widetilde{E}\} .
$$

By construction $J \widetilde{E}=0$. This yields $J \operatorname{VF}_{\text {alg }}(X, Y) \subseteq E$. Furthermore, by Har77, Ch. II Ex 5.6] we have that $V(J) \subseteq Y$. Recall that $I$ is the ideal of $Y$ and let $J^{\prime}=J \cap I$ so that $V\left(J^{\prime}\right)=Y$. Let now $a_{i}$ be a finite set of generators of $i$. Since $\operatorname{rad}\left(J^{\prime}\right)=I$, we have that there exists $\ell_{i}$ such that $a_{i}^{\ell_{i}} \in J$ for all $i$. Letting $\ell=1+\sum_{i}\left(\ell_{i}-1\right)$ we obtain

$$
I^{\ell} \subseteq J^{\prime} \subseteq J \quad \text { and so } \quad I^{\ell} \operatorname{VF}_{\text {alg }}(X, Y) \subseteq J \operatorname{VF}_{\text {alg }}(X, Y) \subseteq E \subseteq \operatorname{Lie}_{a l g}(X, Y) .
$$

Hence the theorem follows.

\section{The Algebraic DENSITY PROPERTy FOR AFFINE TORIC VARIETIES}

We first recall the basic facts from toric geometry that will be needed in this section. They can be found in any text about toric geometry such as [Ful93, Oda88, CLS11.

Let $M$ and $N$ be mutually dual lattices of rank $n$ with duality pairing $M \times N \rightarrow \mathbb{Z}$, where $(m, p) \mapsto\langle m, p\rangle=p(m)$. We also let $M_{\mathbb{Q}}=M \otimes_{\mathbb{Z}} \mathbb{Q}$ and $N_{\mathbb{Q}}=N \otimes_{\mathbb{Z}} \mathbb{Q}$. Letting $\mathrm{T}$ be the algebraic torus $\mathrm{T}=\operatorname{Spec} \mathbb{C}[M]=N \otimes_{\mathbb{Z}} \mathbb{C}^{*}$. A toric variety is a normal variety endowed with an effective action of T having an open orbit. Since the T-action is effective, the open orbit is equal to $\mathrm{T}$.

It is well known that affine toric varieties can be described by means of strongly convex polyhedral cones (pointed cones) in the vector space $N_{\mathbb{Q}}$. Indeed, let $\sigma$ be a pointed cone in $N_{\mathbb{Q}}$, then $X_{\sigma}=\operatorname{Spec} \mathbb{C}\left[\sigma^{\vee} \cap M\right]$ is an affine toric variety and every affine toric variety arises this way. Here $\mathbb{C}\left[\sigma^{\vee} \cap M\right]$ is the semigroup algebra $\mathbb{C}\left[\sigma^{\vee} \cap M\right]=\bigoplus_{m \in \sigma^{\vee} \cap M} \mathbb{C} \chi^{m}$. In the following, we denote $\sigma^{\vee} \cap M$ by $\sigma_{M}^{\vee}$.

There is a one to one correspondence between the faces $\tau$ of the cone $\sigma$ and the orbits $\mathcal{O}(\tau)$ of the T-action on $X_{\sigma}$ (usually called the Orbit-Cone correspondence). The dimension of an orbit is given by $\operatorname{dim} \mathcal{O}(\tau)=\operatorname{rank} N-\operatorname{dim} \tau$ and its closure is given by $\overline{\mathcal{O}(\tau)}=\bigcup_{\delta} \mathcal{O}(\delta)$ where $\delta$ runs over all faces of $\sigma$ containing $\tau$. The ideal $I(\tau)$ of an orbit closure $\overline{\mathcal{O}(\tau)}$ is given by

$$
I(\tau)=\bigoplus_{m \in \sigma_{M}^{\vee} \backslash \tau^{\perp}} \mathbb{C} \chi^{m}
$$

where $\tau^{\perp} \subseteq M_{\mathbb{Q}}$ is the orthogonal of $\tau$. Furthermore, the ideal of $X \backslash \mathrm{T}$ is

$$
I(X \backslash \mathrm{T})=\bigoplus_{m \in\left(\operatorname{rel} . \text {.int } \sigma^{\vee}\right) \cap M} \mathbb{C} \chi^{m},
$$

where rel. int denotes the relative interior. 
As usual, we identify a ray $\rho \subseteq \sigma$ with its primitive vector. The set of all the rays of $\sigma$ is denoted by $\sigma(1)$. A cone $\sigma$ is called smooth if $\sigma(1)$ is part of a basis of the lattice $N$. Let $\tau \subseteq \sigma$ be any face. The orbit $\mathcal{O}(\tau)$ is contained in $X^{\text {reg }}$ if and only if $\tau$ is smooth.

Let now $e \in M$ and $p \in N$. The linear map

$$
\partial_{e, p}: \mathbb{C}[M] \rightarrow \mathbb{C}[M], \quad \chi^{m} \mapsto\langle m, p\rangle \cdot \chi^{m+e}
$$

is a homogeneous derivation of the algebra $\mathbb{C}[M]$ and so it is a homogeneous vector field on $\mathrm{T}=$ Spec $\mathbb{C}[M]$. By the exponential map, the tangent space of $\mathrm{T}=N \otimes_{\mathbb{Z}} \mathbb{C}^{*}$ at the identity $\mathfrak{e} \in \mathrm{T}$ is isomorphic to $N \otimes_{\mathbb{Z}} \mathbb{C}$ and the evaluation of the vector field $\partial_{e, p}$ at the smooth point $\mathfrak{e}$ is $\partial_{e, p}[\mathfrak{e}]=p$.

Let $\sigma \subseteq N_{\mathbb{Q}}$ be a pointed cone. The following proposition gives a description of all the homogeneous vector fields on $X_{\sigma}$. The first statement of the following result can be found in Dem70. For the convenience of the reader we provide a short argument.

Proposition 3.1. The homogeneous vector field $\partial_{e, p}$ on $\mathrm{T}$ extends to a homogeneous vector field in $X_{\sigma}$ if and only if

Type I: $e \in \sigma_{M}^{\vee}$, or

Type II: There exists $\rho_{e} \in \sigma(1)$ such that

$$
\begin{aligned}
& \text { (a) } p \in \mathbb{Z} \rho_{e} \text {, } \\
& \text { (b) }\left\langle e, \rho_{e}\right\rangle=-1 \text {, and } \\
& \text { (c) }\langle e, \rho\rangle \geq 0 \text { for all } \rho \in \sigma(1) \backslash\left\{\rho_{e}\right\} \text {. }
\end{aligned}
$$

Furthermore, $\partial_{e, p}$ is locally nilpotent if and only if it is of type II, and $\partial_{e, p}$ is semisimple if and only if it is of type $I$ and $e=0$.

Proof. The vector field $\partial_{e, p}$ extends to $X_{\sigma}$ if and only if $\partial_{e, p}\left(\mathbb{C}\left[\sigma_{M}^{\vee}\right]\right) \subseteq \mathbb{C}\left[\sigma_{M}^{\vee}\right]$. Since $\mathbb{C}\left[\sigma_{M}^{\vee}\right]$ is spanned by $\chi^{m}$ for all $m \in \sigma_{M}^{\vee}$, it is enough to show that $\partial_{e, p}\left(\chi^{m}\right) \in \mathbb{C}\left[\sigma_{M}^{\vee}\right]$. In combinatorial terms, this corresponds to the condition:

$$
\text { For every } m \in \sigma_{M}^{\vee} \backslash p^{\perp} \text {, we have }\langle m+e, \rho\rangle \geq 0 \text { for all } \rho \in \sigma(1) \text {. }
$$

Assume first that $p$ is not proportional to any $\rho \in \sigma(1)$. Then for every $\rho \in \sigma(1)$ there exists $m \in \sigma_{M}^{\vee}$ such that $\langle\rho, m\rangle=0$ and $\langle p, m\rangle \neq 0$. Hence, (11) implies that $\langle\rho, e\rangle \geq 0$ and so $\partial_{e, p}$ is of type I.

Assume now that there exists $\rho_{e} \in \sigma(1)$ such that $p \in \mathbb{Z} \rho_{e}$. With the same argument as above we can show that $\langle\rho, e\rangle \geq 0$ for all $\rho \in \sigma(1) \backslash\left\{\rho_{e}\right\}$. Let now $m \in \sigma_{M}^{\vee}$ such that $\left\langle\rho_{e}, m\right\rangle=1$. Then (1) implies that $\left\langle\rho_{e}, m+e\right\rangle \geq 0$. This yields $\left\langle\rho_{e}, e\right\rangle \geq-1$. If $\left\langle\rho_{e}, e\right\rangle=-1$ then $\partial_{e, p}$ is of type II. If $\left\langle\rho_{e}, e\right\rangle>-1$ then $\left\langle\rho_{e}, e\right\rangle \geq 0$ and $\partial_{e, p}$ is of type I.

To prove the second assertion, we let $\partial=\partial_{e, p}$ be a homogeneous vector field. A straightforward computation shows that

$$
\partial^{\ell+1}\left(\chi^{m}\right)=\langle m+\ell e, p\rangle \cdot \partial^{\ell}\left(\chi^{m}\right) \cdot \chi^{e} .
$$

Assume first that $\partial$ is of type I and that $e \in \sigma_{M}^{\vee} \backslash\{0\}$. If $\langle e, p\rangle \neq 0$ then (2) yields

$$
\partial^{\ell}\left(\chi^{e}\right)=\ell ! \cdot\langle e, p\rangle^{\ell} \cdot \chi^{\ell e} \neq 0
$$

and so $\partial$ is not locally finite since $\operatorname{span}\left\{\chi^{k e} \mid k \in \mathbb{Z}_{\geq 0}\right\}$ is not finite dimensional. If $\langle e, p\rangle=0$ then let $m \in \sigma_{M}^{\vee}$ be such that $\langle m, p\rangle \neq 0$. In this case (2) implies

$$
\partial^{\ell}\left(\chi^{m}\right)=\langle m, p\rangle^{\ell} \cdot \chi^{m+(\ell-1) e} \neq 0,
$$

and again $\partial$ is not locally finite with a similar argument.

Assume now that $\partial$ is of type I and that $e=0$. The vector field $\partial$ is the infinitesimal generator of the algebraic $\mathbb{C}^{*}$-action on $X_{\sigma}$ given by the $\mathbb{Z}$-grading on $\mathbb{C}\left[\sigma_{M}^{\vee}\right]$ induced by the degree function $\operatorname{deg}\left(\chi^{m}\right)=\langle p, m\rangle$. Hence, the vector field $\partial$ is semisimple.

Finally, assume that $\partial$ is of type II. For every $m \in \sigma_{M}^{\vee}$ we let $\ell=\left\langle m, \rho_{e}\right\rangle$. Now, $\partial_{e, p}$ is locally nilpotent since $\partial_{e, p}^{\ell+1}\left(\chi^{m}\right)=0$ by (2). 
In the following corollary, we give an explicit description of the homogeneous complete vector fields on an affine toric variety.

Corollary 3.2. The vector field $\partial_{e, p}$ is complete if and only if it is of type II, or it is of type I and $\langle e, p\rangle=0$.

Proof. The vector fields of type II are locally nilpotent, hence complete. In the following, we assume that $\partial=\partial_{e, p}$ is of type I. First, assume that $\langle e, p\rangle=0$. Then $\partial=\chi^{e} \cdot \partial_{0, p}$ and since $\chi^{e}$ belongs to the kernel of $\partial_{0, p}$, we have that $\partial$ is complete.

Assume now that $\langle p, e\rangle \neq 0$. Let $I$ be the ideal of $X \backslash$ T, i.e.,

$$
I=\bigoplus_{m \in \operatorname{rel} . \operatorname{int}\left(\sigma^{\vee}\right) \cap M} \mathbb{C} \chi^{m}
$$

Since $e \in \sigma_{M}^{\vee}$, we have that $\partial(I) \subseteq I$. Hence, $X \backslash \mathrm{T}$ is invariant by $\partial_{e, p}$ and so $\mathrm{T}$ is also invariant by $\partial_{e, p}$. In the following, we show that $\partial$ is not complete when restricted to T. Since $\lambda \partial, \lambda \in \mathbb{C}^{*}$ is complete if and only if $\partial$ is complete, we will assume that $p$ is a primitive vector in $N$ and $\langle e, p\rangle>0$.

Without loss of generality, we choose mutually dual bases of $N$ and $M$ such that $p=$ $(1,0, \ldots, 0)$ and $e=\left(e_{1}, \ldots, e_{n}\right)$, with $e_{1}>0$ and $n=\operatorname{rank} N$. We will also denote $x_{i}=\chi^{\beta_{i}}$ the standard coordinates of the torus $\mathrm{T}$, where $\left\{\beta_{i} \mid i=1, \ldots, n\right\}$ is the base of $N$. In this coordinates, the vector field $\partial$ restricted to $\mathrm{T}$ is given by

$$
\partial=x_{1}^{e_{1}+1} x_{2}^{e_{2}} \cdots x_{n}^{e_{n}} \frac{\partial}{\partial x_{1}},
$$

which is not complete on $\mathrm{T}$ since $e_{1}>0$. Indeed the vector fields $x^{n} \partial / \partial x$ on $\mathbb{C}$ are not complete for $n \geq 2$.

Remark that in Corollary 3.2 complete vector fields of type I are extensions of complete vector fields on the big torus $\mathrm{T}$ while complete vector fields of type II are locally nilpotent, hence not complete in $\mathrm{T}$. In the next lemma, we give a criterion for a homogeneous vector field to vanish in an orbit closure.

Lemma 3.3. Let $\partial_{e, p}$ be a non-zero homogeneous vector field on $X_{\sigma}$ and let $\tau \subseteq \sigma$ be a face. Then $\partial_{e, p}$ vanishes at the orbit closure $\overline{\mathcal{O}(\tau)}$ if and only if

Type I: $p \in \operatorname{Span} \tau$ or $\langle e, \rho\rangle>0$ for some $\rho \in \tau(1)$.

Type II: $\langle e, \rho\rangle>0$ for some $\rho \in \tau(1)$.

Proof. The vector field $\partial_{e, p}$ does not vanish at the orbit closure $\overline{\mathcal{O}(\tau)}$ if and only if $\partial_{e, p}\left(\mathbb{C}\left[\sigma_{M}^{\vee}\right]\right) \nsubseteq$ $I(\tau)$. In combinatorial terms this happens if and only if

$$
\text { there exists } m \in \sigma_{M}^{\vee} \backslash p^{\perp} \text { such that }\langle m+e, \rho\rangle=0 \text { for all } \rho \in \tau(1) \text {. }
$$

Case of type I. In this case, we have $e \in \sigma_{M}^{\vee}$ so $\langle m+e, \rho\rangle=0$ for all $\rho \in \tau(1)$ if and only if $\langle m, \rho\rangle=0$ and $\langle e, \rho\rangle=0$ for all $\rho \in \tau(1)$. This is the case if and only if $m \in \tau^{\perp}$ and $e \in \tau^{\perp}$. Such and $m \in \sigma_{M}^{\vee} \backslash p^{\perp}$ exists if and only if $\tau^{\perp} \nsubseteq p^{\perp}$, i.e., if and only if $p \notin \operatorname{Span} \tau$. Hence, we conclude that $\partial_{e, p}$ does not vanish at the orbit closure $\overline{\mathcal{O}(\tau)}$ if and only if $p \notin \operatorname{Span} \tau$ and $\langle e, \rho\rangle=0$ for all $\rho \in \tau(1)$.

Case of type II. In this case we have that there exists $\rho_{e} \in \sigma(1)$ such that $p \in \mathbb{Z} \rho_{e} \backslash\{0\}$, $\left\langle e, \rho_{e}\right\rangle=-1$, and $\langle e, \rho\rangle \geq 0$ for all $\rho \in \sigma(1) \backslash\left\{\rho_{e}\right\}$.

Assume first that $\rho_{e} \notin \tau(1)$. An argument similar to case I yields that $\partial_{e, p}$ does not vanish at the orbit closure $\overline{\mathcal{O}(\tau)}$ if and only if $p \notin \operatorname{Span} \tau$ and $\langle e, \rho\rangle=0$ for all $\rho \in \tau(1)$. Since $\rho_{e} \notin \tau(1)$, we have that $p \notin \operatorname{Span} \tau$ and so the vector field $\partial_{e, p}$ does not vanish at the orbit closure $\overline{\mathcal{O}(\tau)}$ if and only if $\langle e, \rho\rangle=0$ for all $\rho \in \tau(1)$.

Assume now that $\rho_{e} \in \tau(1)$. If there exists $\rho \in \tau(1)$ such that $\langle e, \rho\rangle>0$, then $\langle m+e, \rho\rangle>0$ for all $m \in \sigma_{M}^{\vee}$ and so $\partial_{e, p}$ vanishes at the orbit $\overline{\mathcal{O}(\tau)}$ by (3). Assume $\langle e, \rho\rangle=0$ for all 
$\rho \in \tau(1) \backslash\left\{\rho_{e}\right\}$ and let $m \in \sigma_{M}^{\vee}$ be such that $\left\langle m, \rho_{e}\right\rangle=1$ and $\langle m, \rho\rangle=0$ for all $\rho \in \tau(1) \backslash\left\{\rho_{e}\right\}$. We have $\left\langle m, \rho_{e}\right\rangle \neq 0$ so $m \notin p^{\perp}$ and $\langle m+e, \rho\rangle=0$ for all $\rho \in \tau(1)$. By (3), we conclude that $\partial_{e, p}$ does not vanish at the orbit closure $\overline{\mathcal{O}(\tau)}$.

Remark 3.4. The degree of a homogeneous locally nilpotent vector fields (of type II) is called a root of $\sigma$. The set of all roots of $\sigma$ is denoted by $\mathcal{R}(\sigma)$. For a root $e \in \mathcal{R}(\sigma)$, the ray $\rho_{e}$ is called the distinguished ray of $e$ and the $\mathbb{G}_{\mathrm{a}}$-action generated by the homogeneous locally nilpotent vector field $\partial_{e, \rho_{e}}$ is denoted by $H_{e}$.

In order to show the ADP for toric varieties, we need to show that $X_{\sigma}$ is homogeneous with respect to some closed subvariety $Y$. In [AZK12, the authors prove that $X_{\sigma}$ is homogeneous with respect to $X_{\sigma}^{\text {sing }}$. In fact, they show that the group of special automorphisms acts infinitetransitively with respect to $X_{\sigma}^{\text {sing }}$. In the following, we will show how their methods can be applied to show that $X_{\sigma}$ is homogeneous with respect to any T-invariant closed subvariety $Y$.

Proposition 3.5. Let $\sigma \subseteq N_{\mathbb{Q}}$ be a pointed cone and let $Y$ be any $\mathrm{T}$-invariant closed subvariety of $X_{\sigma}$ containing $X_{\sigma}^{\text {sing }}$. Then $X_{\sigma}$ is homogeneous relative to $Y$.

Proof. Using the T-action and the Orbit-Cone correspondence, to prove the theorem it is enough to find, for every orbit $\mathcal{O}(\tau)$ in $X_{\sigma}^{\mathrm{reg}}$ different from the open orbit, an automorphism that

(i) sends a point $x$ in $\mathcal{O}(\tau)$ into an orbit of higher dimension, and

(ii) leaves stable every orbit not containing $\mathcal{O}(\tau)$ in its closure.

Let $\rho_{1}, \ldots, \rho_{\ell}$ be the rays of $\tau$. In [AZK12, Lemma 2.3] and its proof, the authors show that for every smooth orbit $\mathcal{O}(\tau)$ there exists a root $e \in \mathcal{R}(\sigma)$ such that

$$
\left\langle\rho_{1}, e\right\rangle=-1,\left\langle\rho_{2}, e\right\rangle=\ldots=\left\langle\rho_{\ell}, e\right\rangle=0 \text {, and }\langle\rho, e\rangle>0 \text { for all rays } \rho \notin \tau(1) .
$$

Furthermore, they show that a generic automorphism $\alpha$ in the $\mathbb{G}_{\mathrm{a}}$-action $H_{e}$ corresponding to the root $e$ satisfies $(i)$.

Let $\mathcal{O}(\delta)$ be any orbit that does not contain $\mathcal{O}(\tau)$ in its closure. In combinatorial terms, this means that $\delta$ is a face of $\sigma$ that is not contained in $\tau$. We claim that $H_{e}$ leaves $\overline{\mathcal{O}(\delta)}$ point-wise invariant and so $\alpha$ satisfies (ii) which proves the proposition.

In terms of the vector field $\partial_{e, \rho_{e}}$, our claim is equivalent to $\partial_{e, \rho_{e}}$ vanishes at $\overline{\mathcal{O}(\delta)}$. Since $\delta$ is not contained in $\tau$ there exists a ray $\rho$ of $\delta$ that is not a ray of $\tau$. By (4) we have $\langle e, \rho\rangle>0$. Now the claim follows from Lemma 3.3 .

For our next theorem we need the following lemma that follows by direct computation.

Lemma 3.6. Let $\partial_{e_{1}, p_{1}}$ and $\partial_{e_{2}, p_{2}}$ be two homogeneous vector fields. Then $\left[\partial_{e_{1}, p_{1}}, \partial_{e_{2}, p_{2}}\right]=\partial_{e, p}$, where $p=p_{1}\left(e_{2}\right) \cdot p_{2}-p_{2}\left(e_{1}\right) \cdot p_{1}$ and $e=e_{1}+e_{2}$.

Theorem 3.7. Let $X$ be a affine toric variety of dimension at least two and let $Y$ be a Tinvariant closed subvariety of $X$ containing $X^{\text {sing }}$. Then $X$ has the ADP relative to $Y$ if and only if $X \backslash Y \neq \mathrm{T}$.

Proof. Let $X=X_{\sigma}$ be the toric variety given by the pointed cone $\sigma \in N_{\mathbb{Q}}$ and let $X_{\sigma} \backslash Y \neq \mathrm{T}$. There is at least one codimension one T-orbit not contained in $Y$. Assume it is $\mathcal{O}\left(\rho_{1}\right)$ for some ray $\rho_{1} \in \sigma(1)$. Let $e_{1}$ be a root with $\rho_{1}$ as distinguished ray. By (44), we can assume that $\left\langle e_{1}, \rho\right\rangle>0$ for all $\rho \in \sigma(1) \backslash\left\{\rho_{1}\right\}$. By Lemma 3.3, the locally nilpotent vector field $\partial_{e_{1}, \rho_{1}}$ vanishes at $Y$ and so $\partial_{e_{1}, \rho_{1}} \in \operatorname{VF}_{\text {alg }}\left(X_{\sigma}, Y\right)$.

Letting $e_{2}, e_{3} \in \operatorname{rel} \operatorname{int}\left(\sigma^{\vee}\right) \cap M$ be such that $e_{3}=e_{1}+e_{2}$, we let

$$
L=\operatorname{Span}\left\{\partial_{e, p} \mid p \in N, e \in e_{3}+\sigma_{M}^{\vee}\right\} .
$$

The set $L$ is contained in $\mathrm{VF}_{\text {alg }}\left(X_{\sigma}, Y\right)$ since $\partial_{e, p} \in L$ vanishes in $X_{\sigma} \backslash \mathrm{T}$. In fact, $L$ is a submodule of $\mathrm{VF}_{\text {alg }}\left(X_{\sigma}, Y\right)$ since for every $m \in \sigma_{M}^{\vee}$ and every $\partial_{e, p} \in L$, we have $\chi^{m} \partial_{e, p}=$ 
$\partial_{e+m, p} \in L$. Furthermore, the fiber over the identity $\mathfrak{e} \in \mathrm{T} \subseteq X_{\sigma}$ is given by

$$
L_{\mathfrak{e}}=\operatorname{Span}\left\{\partial_{e, p}[\mathfrak{e}] \mid \partial_{e, p} \in L\right\}=\operatorname{Span}\left\{p \mid \partial_{e, p} \in L\right\}=N \otimes_{\mathbb{Z}} \mathbb{C}=T_{\mathfrak{e}} X_{\sigma},
$$

and so $L_{\mathfrak{e}}$ contains a generating set. We claim that $L \subseteq \operatorname{Lie}_{\text {alg }}\left(X_{\sigma}, Y\right)$. Hence $X_{\sigma}$ has the ADP relative to $Y$ by Theorem 2.2 and Proposition 3.5 .

By Corollary [3.2, the vector field $\partial_{e, p}$ is complete if $\langle e, p\rangle=0$. Hence, to prove our claim it is enough to show that for every $e \in e_{3}+\sigma_{M}^{\vee}$, there exists $p \in N$ such that $\langle e, p\rangle \neq 0$ and $\partial_{e, p} \in \operatorname{Lie}_{\text {alg }}\left(X_{\sigma}, Y\right)$.

Indeed, let $e_{4}=e-e_{1}$ and choose $p_{4}$ be such that $\left\langle e_{4}, p_{4}\right\rangle=0$ and $\left\langle e_{1}, p_{4}\right\rangle \neq 0$ which implies that $\partial_{e_{4}, p_{4}}$ belongs to $\operatorname{Lie}_{\text {alg }}\left(X_{\sigma}, Y\right)$. This is possible since $e_{4}$ lies in rel.int $\sigma^{\vee}$ and $e_{1}$ is a root of $\sigma^{\vee}$. By Lemma 3.6 we have

$$
\left[\partial_{e_{1}, \rho_{1}}, \partial_{e_{4}, p_{4}}\right]=\partial_{e, p} \quad \text { where } \quad p=\rho_{1}\left(e_{4}\right) \cdot p_{4}-p_{4}\left(e_{1}\right) \cdot \rho_{1} .
$$

A routine computation shows that

$$
\langle e, p\rangle=\left\langle e, \rho_{1}\left(e_{4}\right) \cdot p_{4}-p_{4}\left(e_{1}\right) \cdot \rho_{1}\right\rangle=\left\langle e_{1}, p_{4}\right\rangle \neq 0,
$$

proving the claim.

Assume now that $X \backslash Y=\mathrm{T}$. The converse of the theorem follows from the fact that for all affine toric varieties $X$ and all $\ell \in \mathbb{Z}_{>0}$ there is a vector field $\partial \in I^{\ell} \operatorname{VF}_{\text {alg }}(X, X \backslash \mathrm{T}) \backslash \operatorname{Lie}_{\text {alg }}(X, X \backslash$ $\mathrm{T})$, where $I=I(X \backslash \mathrm{T})$. Indeed, Andersén And00 proved that any complete algebraic vector field on $\mathrm{T}$ does preserve the Haar form

$$
\omega=\frac{d x_{1}}{x_{1}} \wedge \ldots \wedge \frac{d x_{n}}{x_{n}} .
$$

Thus if we find $\partial$ in $I^{\ell} \mathrm{VF}_{\text {alg }}(X, X \backslash \mathrm{T})$ whose restriction to $\mathrm{T}$ does not preserve $\omega$ we are done.

After a change of coordinates one can assume that $(1,0, \ldots, 0) \in \operatorname{rel}$. int $\sigma^{\vee}$. Then $\partial=x_{1}^{N} \frac{\partial}{\partial x_{1}}$ is a regular vector field on $X$ contained in $I^{\ell} \mathrm{VF}_{\text {alg }}(X, X \backslash \mathrm{T})$ for $N$ big enough which does not preserve $\omega$.

Remark 3.8. Lárusson proved in [Lár11, For13, that all smooth toric varieties are Oka-Forstnerič manifolds, however it is still unknown if they are elliptic, see [For11, Kut14 for definitions. The proof of Theorem 3.7 can be adapted to prove the following: every smooth quasi-affine toric variety is elliptic (and thus an Oka-Forstnerič manifold). Indeed, the torus $\mathrm{T}$ is well known to be elliptic. Let $X_{0}$ be a smooth quasi-affine toric variety different from T. Let also $X$ be an affine toric variety such that $X_{0} \subseteq X$ is an equivariant open embedding and let $Y=X \backslash X_{0}$. Now, Proposition 3.5 and (5) imply that $X_{0}$ is elliptic [For11, Example 5.5.13 (B)].

\section{Classification of COMplete Vector Fields on AFFine toric SuRfaces}

In this section we classify all complete algebraic vector fields on a given affine toric surface $X_{\sigma}$. The classification works essentially the same as the classification of complete vector fields on $\mathbb{C}^{2}$ done by Brunella Bru04.

From now on we will use the fact that each affine toric surface different from $\mathbb{C}^{*} \times \mathbb{C}$ or $\mathbb{C}^{*} \times \mathbb{C}^{*}$ can be seen as the quotient of $\mathbb{C}^{2}$ by the action of a cyclic group. Let $d$ be the order of the group and let $e$ be a co-prime number $0<e<d$ and consider the action of $\mathbb{Z}_{d}$ given by $\zeta \cdot(u, v)=\left(\zeta u, \zeta^{e} v\right)$ where $\zeta$ is a primitive $d$-th root of unity. We obtain the projection $\pi: \mathbb{C}^{2} \rightarrow \mathbb{C}^{2} / \mathbb{Z}_{d}=: V_{d, e}$ onto our toric surface which is a ramified covering of $V_{d, e}$ ramified only over the unique singular point. Certainly each vector field on $X$ pulls back to an invariant vector field of $\mathbb{C}^{2}$ by using the fiber-wise isomorphism $D \pi$ on the tangent space. A complete vector field on $V_{d, e}$ will pull back to an invariant complete vector field on $\mathbb{C}^{2}$.

Definition 4.1. Let $f: \mathbb{C}^{2} \rightarrow \mathbb{C}$ be a regular function on $\mathbb{C}^{2}$. The function $f$ is called $\mathbb{Z}_{d^{-}}$ preserved if the fibers of $f$ are sent to fibers of $f$ by the $\mathbb{Z}_{d}$-action. It is called $\mathbb{Z}_{d}$-homogeneous of degree $[i] \in \mathbb{Z}_{d}$ if $\zeta^{*} f(u, v)=f(\zeta \cdot(u, v))=\zeta^{i} f(u, v)$ for all $(u, v) \in \mathbb{C}^{2}$. Let $A_{[i]}$ denote the 
space of $\mathbb{Z}_{d}$-homogeneous polynomials of degree $[i]$ then we obtain a decomposition of the ring of regular functions on $\mathbb{C}^{2}$ into $\mathbb{Z}_{d}$-homogeneous parts $\mathbb{C}[u, v]=A_{[0]} \oplus \ldots \oplus A_{[d-1]}$. In particular $A_{[0]}$ is the ring of invariant functions $\mathbb{C}[u, v]^{\mathbb{Z}_{d}}=\mathbb{C}\left[V_{d, e}\right]$.

It is clear from the definition that $A_{[i]}$ is spanned by all monomials $u^{m} v^{n}$ with $[m+e n]=[i] \in$ $\mathbb{Z}_{d}$. Clearly invariant vector fields are of the form $f \partial / \partial u+g \partial / \partial v$ with $f \in A_{[1]}$ and $g \in A_{[e]}$. Moreover we have the following easy lemma:

Lemma 4.2. Let $f: \mathbb{C}^{2} \rightarrow \mathbb{C}$ be a regular function then the following are equivalent:

(1) $f$ is $\mathbb{Z}_{d}$-homogeneous,

(2) $f$ is $\mathbb{Z}_{d}$-preserved with $f(0,0)=0$,

(3) $f^{-1}(0)$ is $\mathbb{Z}_{d^{-}}$invariant.

Proof. (1) implies (2) since if $f$ is constant on a curve then also $\zeta^{i} \cdot f$ is constant and $f(0,0)=0$ follows directly from the homogeneity. The fiber $f^{-1}(0)$ contains the $\mathbb{Z}_{d}$-fixed point $(0,0)$ thus $(3)$ follows from (2). If the zero fibers of $f$ and $\zeta^{*} f$ coincide then we have that $\zeta^{*} f(u, v)=a \cdot f(u, v)$ for some $a \in \mathbb{C}^{*}$. By $f(u, v)=\zeta^{d *} f(u, v)=a^{d} f(u, v)$ we see that $a$ is a $d$-th root of unity and thus (3) implies (1).

The following lemma is the crucial step in the classification of invariant complete algebraic vector fields and hence of complete algebraic vector fields on the toric variety $V_{d, e}$. Recall that a rational first integral of a vector field is a rational function such that its fibers are tangential to the vector field.

Lemma 4.3. Let $\partial$ be a $\mathbb{Z}_{d}$-invariant complete algebraic vector field on $\mathbb{C}^{2}$ then $\partial$ preserves either a $\mathbb{Z}_{d}$-homogeneous fibration $f: \mathbb{C}^{2} \rightarrow \mathbb{C}$ with general fibers $\mathbb{C}$ or $\mathbb{C}^{*}$ or $\partial$ has a reduced rational first integral $g: \mathbb{C}^{2} \rightarrow \mathbb{C}$.

Proof. By Bru04 there is fibration $f: \mathbb{C}^{2} \rightarrow \mathbb{C}$ with $\mathbb{C}$ or $\mathbb{C}^{*}$ fibers which is preserved by the flow $\varphi^{t}$ of $\partial$. We may assume that $f(0,0)=0$. If $f$ is $\mathbb{Z}_{d}$-homogeneous then we are done. If $f$ is not

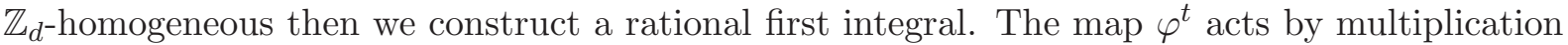
with some $a_{t}$ on the set of fibers of $f$ parametrized by $\mathbb{C}$ so we have $f\left(\varphi^{t}(u, v)\right)=a_{t} f(u, v)$ (indeed $(0,0)$ is a fixed point of $\left.\varphi^{t}\right)$. Since $\partial$ is invariant the same holds true for $g(u, v)=f(\zeta \cdot(u, v))$ and hence the rational map $f / g$ is a rational first integral for $\partial$. By Stein factorization $\partial$ has a reduced first integral. Recall that every rational function $\mathbb{C}^{2} \rightarrow \mathbb{P}^{1}$ can be decomposed into $F \circ \tilde{f} \mathbb{C}^{2} \rightarrow \mathbb{P}^{1} \rightarrow \mathbb{P}^{1}$ such that $\tilde{f}$ has connected regular fibers, or equivalently is reduced. This factorization is called Stein factorization.

The next step will be the classification of $\mathbb{Z}_{d^{-}}$homogeneous fibrations with general fibers $\mathbb{C}$ or $\mathbb{C}^{*}$ and rational first integrals for invariant vector fields. The classification will be done up to equivariant automorphisms of $\mathbb{C}^{2}$ which will lead to a classification of the vector fields on $V_{d, e}$ up to automorphism of $V_{d, e}$ since equivariant automorphisms clearly project down to automorphims of the quotient. Equivariant automorphisms of $\mathbb{C}^{2}$ are given by invertible maps $(u, v) \mapsto(p(u, v), q(u, v))$ with $p \in A_{[1]}$ and $q \in A_{[e]}$.

First we establish an equivariant version of the Abhyankar-Moh Theorem. We provide a proof using the classical verion of the theorem. See AZ13 for a different proof.

Lemma 4.4. Let $\mathbb{C} \cong L \subset \mathbb{C}^{2}$ be a line which is invariant by the group action. Then there is an equivariant automorphism of $\mathbb{C}^{2}$ mapping $L$ to $\{u=0\}$ or $\{v=0\}$. Moreover a cross of two invariant lines can be mapped to $\{u v=0\}$.

Proof. By the classical Abhyankar-Moh Theorem we know that $L$ is given by a polynomial $p$ which is a component of an automorphism of $\mathbb{C}^{2}$. In order to find the other component of the automorphism we have to find an invariant section of the trivial line bundle given by $p$. We start with an arbitrary trivialization and get an invariant section taking the average over images of the zero section by the group action. Each image is another section because the action sends 
fibers of $p$ to fibers of $p$ since the zero fiber is invariant. We denote the polynomial giving this invariant section by $q$. The map given by $(p, q)$ is an automorphism of $\mathbb{C}^{2}$ since it is the composition of the trivialization we started with and the map $(u, v) \mapsto(u, v-s(u))$ where $s$ is the invariant section. Because the zero sets of $p$ and $q$ are invariant they are $\mathbb{Z}_{d}$-homogeneous by Lemma 4.2 and since they are the two components of an automorphism their homogeneity degrees coincide with [1] and [e] so either $(p, q)$ or $(q, p)$ is an equivariant automorphism and the claim follows. The second statement is trivial since there we already have an invariant section by assumption.

We get the following corollary as an immediate consequence, see also [FKZ08].

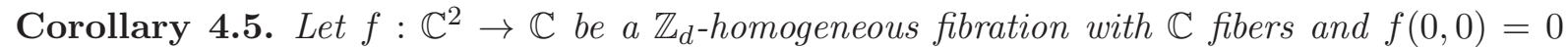
then $f(u, v)=u$ or $f(u, v)=v$ up to equivariant automorphism of $\mathbb{C}^{2}$.

For the classification of $\mathbb{Z}_{d^{-}}$homogeous fibration with $\mathbb{C}^{*}$ fibers we first state the non-equivariant version used in [Bru04, see also [Suz77].

Lemma 4.6. Let $f: \mathbb{C}^{2} \rightarrow \mathbb{C}$ be a fibration with $\mathbb{C}^{*}$ fibers then $f(x, y)$ has one special fiber (say $f^{-1}(0)$ ) and it is isomorphic to $\mathbb{C} \cup \mathbb{C}^{*}$ or $\{x y=0\}$ and $f$ is up to automorphism of $\mathbb{C}^{2}$ of the form $f(x, y)=x^{m}\left(x^{l} y+p(x)\right)^{n}$ or $f(x, y)=x^{m} y^{n}$ for coprime $m, n \in \mathbb{N}, \operatorname{deg} p<l \geq 1$ and $p(0) \neq 0$.

The equivariant version of this lemma is given by the two following lemmas.

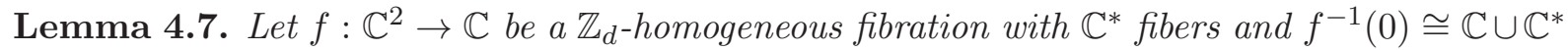
then there are coprime $m, n \in \mathbb{N}$ and an invariant polynomial $p$ with $\operatorname{deg} p<l \geq 1$ and $p(0) \neq 0$ such that up to equivariant automorphism $f(u, v)=u^{m}\left(u^{l} v+p(u)\right)^{n}$ with $[l+e]=[0]$ or $f(u, v)=v^{m}\left(v^{l} u+p(v)\right)^{n}$ with $[1+l e]=[0]$.

Proof. By Lemma 4.6 we know that there exists a not necessary equivariant automorphism $(x(u, v), y(u, v))$ such that $f(x, y)$ is as in Lemma 4.6. Clearly, the curve $\mathbb{C} \cong C \subset f^{-1}(0)$ is invariant by the group action since it is the only fiber component isomophic to $\mathbb{C}$. By Lemma 4.4 we may assume the $C=\{u=0\}$ or $C=\{v=0\}$. In the first case this implies that, up to equivariant automorphism, $x(u, v)=a u$ and $y(u, v)=b v+q(u)$ for some $a, b \in \mathbb{C}^{*}$ and $q \in \mathbb{C}[u]$ and hence $f$ is of the form $(a u)^{m}\left((a u)^{l}(b v+q(u))+p(u)\right)^{n}$ with $\operatorname{deg} p<l$. Since $f$ is $\mathbb{Z}_{d}$-homogeneous we have $q \in A_{[e]}$ and $p \in A_{[l+e]}$ hence the map $(x(u, v), y(u, v))$ was equivariant after all and $f$ has the desired standard form up to equivariant automorphism. The equality $[l+e]=[0]$ follows from the fact that $p(0) \neq 0$. The case $C=\{v=0\}$ leads similarly to the second possibility.

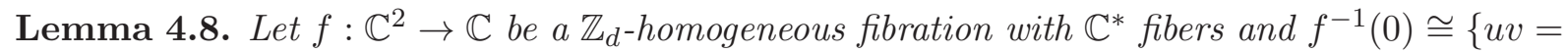
$0\}$ then there are coprime $m, n \in \mathbb{N}$ such that $f(u, v)=u^{m} v^{n}$ up to equivariant automorphism. If $d$ is divisible by $4\left(\right.$ say $\left.d=4 d^{\prime}\right)$ and $e=2 d^{\prime}+1$ then $f$ can also be of the form $f(u, v)=u^{2}-v^{2}$.

Proof. By Lemma 4.6 there is an automorphism $(x(u, v), y(u, v))$ such that $f=x^{m} y^{n}$. Clearly the 0-fiber $\{x(u, v)=0\} \cup\{y(u, v)=0\}$ is invariant by the group action. If the two lines are invariant themselves then by Lemma 4.4 we may assume that they coincide with $\{u v=0\}$ and hence we may assume $x(u, v)=a u$ and $y(u, v)=b v$ for some $a, b \in \mathbb{C}^{*}$ and we are done. If the two lines are interchanged by the group action then we have $d=2 d_{0}$ is even and

$$
x(\zeta \cdot(u, v))=a y(u, v) \quad \text { and } \quad y(\zeta \cdot(u, v)=b x(u, v)
$$

for some $a, b \in \mathbb{C}^{*}$. After rescaling we may assume that $a=b$. The fibration $f=x^{m} y^{n}$ is $\mathbb{Z}_{d}$-homogeneous so $x^{m} y^{n}=$ const $\cdot y^{m} x^{n}$ and hence $m=n=1$. Moreover we have $x(u, v)=$ $x\left(\zeta^{d} \cdot(u, v)\right)=a^{d} x(u, v)$ and hence $a=\zeta^{i}$ for some $i$. We see that the maps $P_{ \pm}(u, v)=$ $x(u, v) \pm y(u, v)$ are $\mathbb{Z}_{d}$-homogeneous and since they are the components of an automorphism of $\mathbb{C}^{2}$ we may assume that the functions $P_{ \pm}$coincides with the functions $u$ and $v$. Altogether we 
have $\frac{1}{4} f(u, v)=(u+v)(u-v)=u^{2}-v^{2}$ which is $\mathbb{Z}_{d}$-homogeneous only if $2 e=2$ or $2 e=2 d_{0}+2$. In the first case $(x(u, v), y(u, v))$ is already equivariant so only the latter case remains. Since $d$ is even and thus $e=d_{0}+1$ is odd we have that $d_{0}=2 d^{\prime}$ is even.

Lemma 4.9. Let $f: \mathbb{C}^{2} \rightarrow \mathbb{P}^{1}$ be a reduced rational first integral of an invariant complete vector field $\partial$ on $\mathbb{C}^{2}$ then up to equivariant automorphism of $\mathbb{C}^{2}$ and Möbius transform of $\mathbb{P}^{1}$ the rational function $f$ is a $\mathbb{Z}_{d}$-homogeneous polynomial with $\mathbb{C}$ or $\mathbb{C}^{*}$ fibers or there are coprime $m, n \in \mathbb{N}$ such that $f(u, v)=u^{m} / v^{n}$.

Proof. A general fiber of $f$ is an orbit closure of the flow of $\partial$. Since $\partial$ is invariant the set of orbits is preserved by the $\mathbb{Z}_{d}$-action hence general fibers of $f$ are mapped to general fibers of $f$ by the action and the action induces a $\mathbb{Z}_{d^{-}}$action on the base $\mathbb{P}^{1}$. Altogether this means that $f$ is $\mathbb{Z}_{d}$-preserved. If $f$ is not surjective then $f$ can be seen as a polynomial which is $\mathbb{Z}_{d}$-homogeneous by Lemma 4.2 and has general fibers isomomorphic $\mathbb{C}$ or $\mathbb{C}^{*}$ since they are orbit closures.

Now consider the case $f$ surjective. As mentioned in [Bru04] and [Suz77] such a first integral is always of the form $f=x^{m} / y^{n}$ for some automorphism $(x(u, v), y(u, v))$. The $\mathbb{Z}_{d}$-action on the base $\mathbb{P}^{1}$ is either trivial (and hence $f$ is $\mathbb{Z}_{d}$-invariant) or it has exactly two fixed points

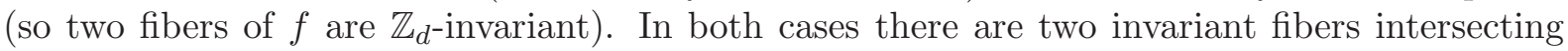
transversally (say the 0 - and the $\infty$-fiber). Indeed if $m=n=1$ all fibers intersect transversely and if $m \neq n$ all but one fiber intersect pairwise tangentially so this fiber is clearly invariant and it intersects all other fibers transversally. By Lemma 4.4 we may assume that these two fibers coincides with $\{u=0\}$ and $\{v=0\}$ and hence $x(u, v)=a u$ and $y(u, v)=b v$ or vice versa.

Theorem 4.10. Let $\partial$ be a complete algebraic vector field on $\mathbb{C}^{2}$ which is invariant by the group action given by $\zeta \cdot(u, v)=\left(\zeta u, \zeta^{e} v\right)$ where $\zeta$ is a primitive $n$-th root of unity and $0<e<d$ coprime numbers. Then $\partial$ has, up to equivariant automorphism of $\mathbb{C}^{2}$, one of the forms in the following list.
(a) $\partial=a u \frac{\partial}{\partial u}+\left(\left(A\left(u^{d}\right) v+B\left(u^{e}\right)\right) \frac{\partial}{\partial v}\right.$
(b) $\partial=a v \frac{\partial}{\partial v}+\left(\left(A\left(v^{d}\right) u+B\left(v^{e^{\prime}}\right)\right) \frac{\partial}{\partial u}\right.$

with $a \in \mathbb{C}, 0<e^{\prime}<d$ such that $\left[e e^{\prime}\right]=[1] \in \mathbb{Z}_{d}$ and $A, B \in \mathbb{C}[t]$.

(a) $\partial=a v \frac{\partial}{\partial v}+A\left(u^{m} v^{n}\right)\left[n u \frac{\partial}{\partial u}-m v \frac{\partial}{\partial v}\right]$

(b) If $d=4 d^{\prime}$ and $e=2 d^{\prime}+1$ then we also have

$$
\partial=a(u+v)\left(\frac{\partial}{\partial u}+\frac{\partial}{\partial v}\right)+A\left(\left(u^{2}-v^{2}\right)^{2 d^{\prime}}\right)\left[u \frac{\partial}{\partial v}+v \frac{\partial}{\partial u}\right]
$$

with $a \in \mathbb{C}, m, n \in \mathbb{N}$ with $[m+e n]=[0]$ and $A \in \mathbb{C}[t]$.

(3) There are $a \in \mathbb{C}, m, n, l \in \mathbb{N}$ with $[m]=[0], p \in A_{[0]}$, $\operatorname{deg} p<l, p(0) \neq 0$ and $A \in \mathbb{C}[t]$ with the property that

$$
A\left(x^{m}\left(x^{l} y+p(x)\right)^{n}\right) \cdot\left(m p(x)+n x p^{\prime}(x)\right)-a p(x) \in x^{l} \cdot \mathbb{C}[x, y]
$$

such that

$$
\begin{aligned}
& \text { (a) } \partial= a\left(v+\frac{p(u)}{u^{l}}\right) \frac{\partial}{\partial v}+ \\
& A\left(u^{m}\left(u^{l} v+p(u)\right)^{n}\right) \cdot\left[n u \frac{\partial}{\partial u}-\left((m+n l) v+\frac{m p(u)+n u p^{\prime}(u)}{u^{l}}\right) \frac{\partial}{\partial v}\right]
\end{aligned}
$$


with $[l+e]=0$.

$$
\begin{aligned}
& \text { (b) } \partial=a\left(u+\frac{p(v)}{v^{l}}\right) \frac{\partial}{\partial u}+ \\
& A\left(v^{m}\left(v^{l} u+p(v)\right)^{n}\right) \cdot\left[n v \frac{\partial}{\partial v}-\left((m+n l) u+\frac{m p(v)+n v p^{\prime}(v)}{v^{l}}\right) \frac{\partial}{\partial u}\right] \\
& \text { with }[1+l e]=0 \text {. }
\end{aligned}
$$

Proof. By Theorem 4.3 we know that the flow $\partial$ preserves fibers of a $\mathbb{Z}_{d}$-homogeneous $\mathbb{C}$ - or $\mathbb{C}^{*}$ polynomial (which are described in Corollary 4.5 and Lemmas 4.7 and 4.8) or it has a rational first integral (which may be assumed to be of the form $u^{m} / v^{n}$ by Lemma 4.9). Once we have a polynomial that is preserved by the flow we can check in Proposition 2 in Bru04 how the vector field looks like. Since the vector fields need to be $\mathbb{Z}_{d}$-invariant some extra conditions are required. In the case of the rational first integral we have $\partial=n u \partial / \partial u+m v \partial / \partial v$ which is already in the list.

\section{The Strong Algebraic DENSity PROPERTy FOR AFFine toriC SURFACES}

First we give a new concept of the ADP which was first introduced in [KK12].

Definition 5.1. Let $\Gamma$ be a group acting on an smooth affine algebraic variety $X$. Then $X$ has $\Gamma$-ADP if the Lie algebra of all $\Gamma$-invariant algebraic vector fields coincides with the Lie algebra generated by all $\Gamma$-invariant complete algebraic vector fields.

As in the section above let $d, e \in \mathbb{Z}$ be two coprime numbers with $0<e<d$ and let $\zeta$ be a

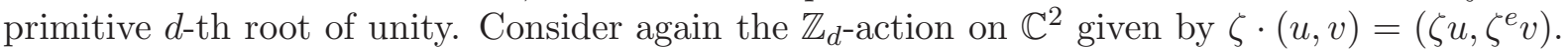
Moreover let $e^{\prime}$ be the unique integer with $0<e^{\prime}<d$ and $e e^{\prime}=1 \bmod d$. It is clear that:

Proposition 5.2. $V_{d, e}$ has the strong $A D P$ if and only if $\mathbb{C}^{2}$ has the $\mathbb{Z}_{d}-A D P$.

Let us introduce the following subsets of $\mathbb{Z}^{2}$

$$
\begin{aligned}
I & =\left\{(i, j) \in \mathbb{Z}_{\geq 0}^{2}: i+e j=0 \bmod d\right\}, \\
J & =\left\{(i, j) \in I \backslash\{(0,0)\}: i<e \text { and } j<e^{\prime}\right\} \subset I,
\end{aligned}
$$

Lemma 5.3. $|J| \leq 1 \Leftrightarrow e \mid d+1$.

Proof. If $e=1$ then also $e^{\prime}=1$ and thus $J=\emptyset$. If $e, e^{\prime}>1$ then $|J| \geq 1$ since $\left(e-1, e^{\prime}-1\right) \in J$. Assume $e e^{\prime}=d+1, i<e$ and $j<e^{\prime}$, then we have $i+j e<e+d<2 d$ and the equality $[i+j e]=[0] \in \mathbb{Z}_{d}$ implies $i+j e=d$. Similarly we get $i e^{\prime}+j=d$ and thus there is a unique solution for $(i, j)$ and hence $|J|=1$. If $e e^{\prime} \geq 2 d+1$ then we get another solution of $[i+j e]=[0]$ in $J$. Indeed, choose $l \in \mathbb{N}$ such that $0<d-l e<e$ then $(d-l e, l) \neq\left(e-1, e^{\prime}-1\right)$ lies in $J$, since $l e<d$ implies $0<l<e^{\prime}-1$.

Let us introduce the following notation:

$$
\begin{aligned}
\mathrm{VF}^{(i, j)} & =\left\{u^{i} v^{j}\left(a u \frac{\partial}{\partial u}+b v \frac{\partial}{\partial v}\right): a, b \in \mathbb{C}\right\} \\
\mathrm{CVF}^{(i, j)} & =\left\{a u^{i} v^{j}\left(j u \frac{\partial}{\partial u}-i v \frac{\partial}{\partial v}\right): a \in \mathbb{C}\right\} \subset \mathrm{VF}^{(i, j)} \\
\mathrm{LND}_{u}^{k} & =\left\{a v^{k e^{\prime}} \frac{\partial}{\partial u}: a \in \mathbb{C}\right\} \\
\mathrm{LND}_{v}^{k} & =\left\{a u^{k e} \frac{\partial}{\partial v}: a \in \mathbb{C}\right\} .
\end{aligned}
$$


Remark that $\mathrm{CVF}^{(i, j)}$ corresponds to the subset of complete vector fields in $\mathrm{VF}^{(i, j)}$ by Corollary 3.2. We have the decomposition of $\mathbb{Z}_{d}$-invariant vector fields in homogeneous vector fields given by:

$$
\mathrm{VF}_{\mathrm{alg}}^{\mathbb{Z}_{d}}\left(\mathbb{C}^{2}\right)=\bigoplus_{(i, j) \in I} \mathrm{VF}^{(i, j)} \oplus \bigoplus_{k \in \mathbb{N}}\left(\mathrm{LND}_{u}^{k} \oplus \mathrm{LND}_{v}^{k}\right)
$$

We define the subspace $S$ of $\operatorname{VF}_{\text {alg }}^{\mathbb{Z}_{d}}\left(\mathbb{C}^{2}\right)$.

$$
S=\bigoplus_{(i, j) \in J} \mathrm{CVF}^{(i, j)} \oplus \bigoplus_{(i, j) \in I \backslash J} \mathrm{VF}^{(i, j)} \oplus \bigoplus_{k \in \mathbb{N}}\left(\mathrm{LND}_{u}^{k} \oplus \mathrm{LND}_{v}^{k}\right)
$$

The following is our main result in this section.

Theorem 5.4. For the Lie algebra $\operatorname{Lie}_{\text {alg }}^{\mathbb{Z}_{d}}\left(\mathbb{C}^{2}\right)$ generated by all $\mathbb{Z}_{d}$-invariant complete algebraic vector fields on $\mathbb{C}^{2}$ we have:

$$
\operatorname{Lie}_{\text {alg }}^{\mathbb{Z}_{d}}\left(\mathbb{C}^{2}\right)= \begin{cases}S & e=e^{\prime} \\ S \oplus\langle\partial\rangle & e \neq e^{\prime}\end{cases}
$$

for any $\partial \in \mathrm{VF}^{\left(e-1, e^{\prime}-1\right)} \backslash \mathrm{CVF}^{\left(e-1, e^{\prime}-1\right)}$. In particular the codimension of the inclusion $\mathrm{Lie}_{\mathrm{alg}}^{\mathbb{Z}_{d}}\left(\mathbb{C}^{2}\right) \subseteq$ $\mathrm{VF}_{\mathrm{alg}}^{\mathbb{Z}_{d}}\left(\mathbb{C}^{2}\right)$ is $|J|$ if $e=e^{\prime}$ and $|J|-1$ otherwise.

Remark that $\operatorname{dim}_{\mathbb{C}} \mathrm{CVF}^{(i, j)}=1$ and $\operatorname{dim}_{\mathbb{C}} \mathrm{VF}^{(i, j)}=2$ as a vector space. Hence, in the case where $e \neq e^{\prime}$ we have that $\mathrm{VF}^{\left(e-1, e^{\prime}-1\right)} \subseteq \operatorname{Lie}_{\text {alg }}^{\mathbb{Z}_{d}}\left(\mathbb{C}^{2}\right)$. We postpone the proof of this theorem to the end of this section.

The theorem immediately shows in which cases $\mathbb{C}^{2}$ has $\mathbb{Z}_{d}$-ADP or, equivalently, $V_{d, e}$ has the strong ADP. It also allows in each particular case to determine the values of $\ell$ from Definition 1.1 for which $I^{\ell} \operatorname{VF}_{\text {alg }}\left(X, X^{\text {sing }}\right) \subseteq \operatorname{Lie}_{\text {alg }}\left(X, X^{\text {sing }}\right)$.

Corollary 5.5. Let $V_{d, e}$ be a toric surface.

(i) $V_{d, e}$ has the strong ADP if and only if if and only if $e \mid d+1$ and $e^{2} \neq d+1$.

(ii) $V_{d, e}$ has the $A D P$ and an upper bound for the minimal $\ell$ such that $I^{\ell} \mathrm{VF}_{\mathrm{alg}}\left(X, X^{\operatorname{sing}}\right) \subseteq$ $\operatorname{Lie}_{\text {alg }}\left(X, X^{\text {sing }}\right)$ is $e+e^{\prime}-2$.

The next lemma shows what is happening if we take the Lie bracket of two complete homogeneous vector fields.

Lemma 5.6. Let $\partial_{1} \in \mathrm{CVF}^{(i, j)}, \partial_{2} \in \mathrm{CVF}^{\left(i^{\prime}, j^{\prime}\right)}, \partial_{3} \in \mathrm{LND}_{u}^{k}$ and $\partial_{4} \in \mathrm{LND}_{v}^{k^{\prime}}$, then

(i) $\left[\partial_{1}, \partial_{2}\right] \in \mathrm{CVF}^{\left(i+i^{\prime}, j+j^{\prime}\right)}$,

(ii) $\left[\partial_{1}, \partial_{3}\right] \in \mathrm{VF}^{\left(i-1, j+k e^{\prime}\right)} \backslash \mathrm{CVF}^{\left(i-1, j+k e^{\prime}\right)}$,

(iii) $\left[\partial_{1}, \partial_{4}\right] \in \mathrm{VF}^{\left(i+k^{\prime} e, j-1\right)} \backslash \mathrm{CVF}^{\left(i+k^{\prime} e, j-1\right)}$,

(iv) $\left[\partial_{3}, \partial_{4}\right] \in \mathrm{VF}^{\left(k^{\prime} e-1, k e^{\prime}-1\right)}$. Furthermore, $\left[\partial_{3}, \partial_{4}\right] \in \mathrm{CVF}^{\left(k^{\prime} e-1, k e^{\prime}-1\right)}$ if and only if $e k^{\prime}=e^{\prime} k$.

Proof. All four statements follow by direct computation using Corollary 3.2 and Lemma 3.6.

The next two lemmas show $\operatorname{Lie}(S)=\operatorname{Lie}_{\text {alg }}^{\mathbb{Z}_{d}}\left(\mathbb{C}^{2}\right)$, each of them showing one inclusion.

Lemma 5.7. $S \subset \operatorname{Lie}_{\text {alg }}^{\mathbb{Z}_{d}}\left(\mathbb{C}^{2}\right)$

Proof. Take $(i, j) \in I \backslash J$, then either $(i-e, j+1) \in I$ or $\left(i+1, j-e^{\prime}\right) \in I$. In the first case pick $\partial \in \mathrm{CVF}^{(i-e, j+1)}$ and $\delta \in \mathrm{LND}_{v}^{1}$ and by Lemma 5.6 we have $[\partial, \delta] \in \mathrm{VF}^{(i, j)} \backslash \mathrm{CVF}^{(i, j)}$ and thus $\mathrm{VF}^{(i, j)} \subset \operatorname{Lie}_{\text {alg }}^{\mathbb{Z}_{d}}\left(\mathbb{C}^{2}\right)$. The second case works similarly.

Lemma 5.8. $\{$ invariant complete algebraic vector fields $\} \subset \operatorname{Lie}(S)$. 
Proof. Let $L$ be the set of vector fields appearing in the list of Theorem 4.10. We will first show that $L \subset S$. Let $\partial \in L$ and $\partial=\sum \partial_{i, j}$ its decomposition into homogeneous parts with respect to the standard grading on $\mathbb{C}^{2}$. We directly see that all homogeneous parts of vector fields (1) and (2a) are complete. For the vector fields $(2 \mathrm{~b})$ and $(3)$ we claim that $\partial_{i, j}=0$ whenever $(i, j) \in J$. Indeed, assume that $\partial_{i, j} \neq 0$ with $(i, j) \neq(0,0)$ and $\partial_{i, j}$ is not an LND. Then in case (2b) we have $e=e^{\prime}=2 d^{\prime}+1, i+j \geq 4 d^{\prime}$ and $i \neq j$ since for every monomial $\mathfrak{m}$ of the polynomial $A$ we have $\operatorname{deg}_{u} \mathfrak{m}-\operatorname{deg}_{v} \mathfrak{m}$ is a multiple of 4 . Hence, either $i>e$ or $j>e^{\prime}$. In case (3a) under the same assumptions we have $i>m+n l-l \geq m \geq d>e$. Similarly, in case (3b) we have $j>m+l n-l \geq m \geq d>e^{\prime}$.

In order to conclude the proof we only need to show that for a vector field $\delta \in \operatorname{Lie}(S)$ and an equivariant automorphism $\phi$ the vector field $\phi_{*} \delta \in \operatorname{Lie}(S)$. By Lemma 4.10 in AZ13 $\phi$ is a composition of equivariant Jonquières automorphisms or more precisely it is a composition of linear equivariant automorphisms and flow maps of the vector fields $u^{k e} \partial / \partial v$ and $v^{k e^{\prime}} \partial / \partial u$ (which are contained in $S$ ). First we show that for any linear automorphism $\phi$ we have $\phi_{*} \delta \in \operatorname{Lie}(S)$. For $e=1$ this statement is true for obvious reasons, indeed here we already have $\operatorname{Lie}(S)=\operatorname{Lie} \mathrm{Z}_{d g}\left(\mathbb{C}^{2}\right)$. For $e \neq 1$ all equivariant linear automorphisms are of the form $(u, v) \mapsto(a u, b v)$ so they act by homothety on homogeneous vector fields of $\operatorname{Lie}(S)$. Now, if $\phi^{t}$ is the flow of the LND $\partial$ then $\phi_{*}^{t} \delta \in \operatorname{Lie}(\partial, \delta)$ for all $t$, since the Taylor expansion of $\phi_{*}^{t} \delta$ gives $\phi_{*}^{t} \delta=\delta+t[\partial, \delta]+\frac{1}{2} t^{2}[\partial,[\partial, \delta]]+$ $\left.\ldots+\frac{1}{n !} t^{n}[\partial, \ldots[\partial, \delta]] \ldots\right]$ which is a finite sum since $\partial$ is an LND and hence its flow is algebraic in $t$. Since $\partial \in S$ the claim follows.

Proof of Theorem 5.4. It is left to show that $\operatorname{Lie}(S)=S$ if $e=e^{\prime}$ and $\operatorname{Lie}(S)=S \oplus\langle\partial\rangle$ if $e \neq e^{\prime}$ for any $\partial \in \mathrm{VF}^{\left(e-1, e^{\prime}-1\right)} \backslash \mathrm{CVF}^{\left(e-1, e^{\prime}-1\right)}$. Let $(i, j) \in J$, then we need to show that $\mathrm{VF}^{(i, j)} \nsubseteq \operatorname{Lie}(S)$ unless $e \neq e^{\prime}$ and $(i, j)=\left(e-1, e^{\prime}-1\right)$. Assume $\operatorname{VF}^{(i, j)} \subset \operatorname{Lie}(S)$, then Lemma [5.6] implies the existence of $\partial \in \mathrm{VF}^{(i, j)} \backslash \mathrm{CVF}^{(i, j)}$ such that $\partial=\left[\partial_{1}, \partial_{2}\right]$ for some $\partial_{1} \in \mathrm{LND}_{u}^{1}$ and $\partial_{2} \in \operatorname{LND}_{v}^{1}, e \neq e^{\prime}$ and $(i, j)=\left(e-1, e^{\prime}-1\right)$.

\section{IMPLICATIONS OF THE ALGEBRAIC DENSITY PROPERTY FOR THE HOLOMORPHIC AUTOMORPHISM GROUP}

We start with the obvious holomorphic version of Definition 1.1, Let $X$ be a Stein space and let $X^{\text {sing }}$ be the singular locus. We also let $Y \subseteq X$ be closed analytic subvariety of $X$ containing $X^{\text {sing }}$ and let $I_{\text {hol }}=I(Y) \subseteq \mathcal{O}(X)$ be the ideal of $Y$. Let $\operatorname{VF}_{\text {hol }}(X, Y)$ be the $\mathcal{O}(X)$-module of holomorphic vector fields vanishing in $Y$ i.e., $\operatorname{VF}_{\text {hol }}(X, Y)=\left\{\partial \mid \partial(\mathcal{O}(X)) \subseteq I_{\text {hol }}\right\}$. Let $\operatorname{Lie}_{\text {hol }}(X, Y)$ be the Lie algebra generated by all the complete vector fields in $\operatorname{VF}_{\text {hol }}(X, Y)$.

Definition 6.1. We say that $X$ has the strong density property (DP) relative to $Y$ if $\operatorname{Lie}_{\text {hol }}(X, Y)$ is dense in $\operatorname{VF}_{\text {hol }}(X, Y)$ in the compact-open topology. Furthermore, we say that $X$ has the DP relative to $Y$ if there exists $\ell \geq 0$ such that $I_{\text {hol }}^{\ell} \operatorname{VF}_{\text {hol }}(X, Y)$ is contained in the closure of $\operatorname{Lie}_{\text {hol }}(X, Y)$. With this definition, the DP relative to $Y$ with $\ell=0$ is just the strong DP relative to $Y$.

Proposition 6.2. Let $X$ be an affine algebraic variety and let $Y$ be a subvariety containing $X^{\text {sing }}$. Then the $A D P$ for $X$ relative to $Y$ implies the $D P$ for $X$ relative to $Y$.

Proof. The proposition follows from the fact that $I^{\ell} \mathrm{VF}_{\text {alg }}(X, Y)$ is dense in $I_{\text {hol }}^{\ell} \mathrm{VF}_{\text {hol }}(X, Y)$. Indeed, by Theorem A of Cartan, there are finitely many global sections $s_{1}, \ldots, s_{N}$ of $I^{\ell} \operatorname{VF}_{\text {alg }}(X, Y)$ that generate the stalk at every point. A standard application of Theorem B of Cartan implies that any holomorphic section $s_{h} \in I_{\text {hol }}^{\ell} \operatorname{VF}_{\text {hol }}(X, Y)$ over an $\mathcal{O}(X)$-convex compact $K \subseteq X$ can be written as $s_{h}=f_{1} s_{1}+\ldots+f_{N} s_{N}$ with $f_{i} \in \mathcal{O}(K)$. By approximating the functions $f_{i}$ by global functions in $\mathbb{C}[X]$, this implies $I^{\ell} \mathrm{VF}_{\text {alg }}(X, Y)$ is dense in $I_{\text {hol }}^{\ell} \mathrm{VF}_{\text {hol }}(X, Y)$. 
Theorem 6.3 (Relative Andersén-Lempert theorem). Let $X$ be a Stein space with the $D P$ relative to a closed analytic subvariety $Y$ containing $X^{\text {sing }}$. Let $\Omega$ be an open subset of $X$. Suppose that $\Phi:[0,1] \times \Omega \rightarrow X$ is a $C^{1}$-smooth map such that

(i) $\Phi_{t}: \Omega \rightarrow X$ is holomorphic and injective for every $t \in[0,1]$,

(ii) $\Phi_{0}: \Omega \rightarrow X$ is the natural embedding of $\Omega$ into $X$,

(iii) $\Phi_{t}(\Omega)$ is a Runge subset of $X$ for every $t \in[0,1]$, and

(iv) $\Phi_{t}(\Omega)$ fixes $Y$ up to order $\ell$, where $\ell$ is such that $I_{\text {hol }}^{\ell} \operatorname{VF}_{\text {hol }}(X, Y)$ is contained the closure of $\operatorname{Lie}_{\text {hol }}(X, Y)$.

Then for each $\epsilon>0$ and every compact subset $K \subset \Omega$ there is a continuous family, $\alpha:[0,1] \rightarrow \operatorname{Aut}_{h o l}(X)$ of holomorphic automorphisms of $X$ fixing $Y$ pointwise such that

$$
\alpha_{0}=i d \text { and }\left|\alpha_{t}-\Phi_{t}\right|_{K}<\epsilon \text { for every } t \in[0,1]
$$

Point (iv) in the assumptions of the theorem means the following: Consider the time dependent vector field $V\left(x, t_{0}\right)=\left.\frac{d}{d t}\right|_{t=t_{0}} \Phi_{t}\left(\Phi_{t_{0}}^{-1}(x)\right)$. The isotopy $\Phi_{t}(\Omega)$ fixes $Y$ up to order $\ell$ if $V\left(x, t_{0}\right)$ is a section of $I_{\text {hol }}^{\ell} \operatorname{VF}_{\text {hol }}(X, Y)$ over $\Phi_{t_{0}}(\Omega)$ for all $t_{0}$.

Sketch of proof. The map $\Phi_{t_{0}}$ is the $t_{0}$-map of the time dependent vector field $V(x, t)$. It can be approximated by dividing the time interval into small pieces and integrating the time independent vector fields over each piece. By assumption, each of those time independent fields is a section in $I_{\text {hol }}^{\ell} \mathrm{VF}_{\text {hol }}(X, Y)\left(\Phi_{t_{0}}(\Omega)\right)$. Since the sheaf $I_{\text {hol }}^{\ell} \mathrm{VF}_{\text {hol }}(X, Y)$ is coherent, a similar use of Theorem A and B of Cartan as in the proof of Proposition 6.2 leads to the fact that these time independent vector fields in the Runge domain $\Phi_{t_{0}}(\Omega)$ can be approximated by global vector fields in $I_{\text {hol }}^{\ell} \mathrm{VF}_{\text {hol }}(X, Y)$. By assumption, these vector fields can be approximated by Lie combinations of complete vector fields vanishing in $Y$ (not necessarily in $I_{\text {hol }}^{\ell} \mathrm{VF}_{\text {hol }}(X, Y)$ ). Now the standard use of Euler's method gives the desired conclusion.

Remark 6.4. If $Y \cap \Phi_{t}(\Omega)=\emptyset$ for all $t \in[0,1]$, then condition (iv) in Theorem 6.3 is trivially satisfied.

Corollary 6.5. Any smooth point in an affine toric variety $X$ of dimension $n \geq 2$ different from the torus has an open neighborhood in the Euclidean topology biholomorphic to $\mathbb{C}^{n}$.

Proof. Let $x \in X$. Take a Runge neighborhood $U$ of $x$ biholomorphic to the unit ball sending $x$ to zero and let $\Phi_{t}$ be the map $\left(1-\frac{t}{2}\right) z$ in the unit ball. Since $X$ has the DP relative to $X^{\text {sing }}$, Theorem 6.3 implies that these contractions can be approximated by holomorphic automorphisms $\alpha_{t}$ of $X$ (fixing $X^{\text {sing }}$ pointwise). The automorphism $\alpha_{1}$ has an attractive fixed point near $x$. The bassin of attraction of this point is biholomorphic to $\mathbb{C}^{n}$ [RR88. Since the holomorphic automorphism group of $X$ is transitive on $X \backslash X^{\text {sing }}$, the claim follows.

\section{REFERENCES}

[AL92] Erik Andersén and László Lempert. On the group of holomorphic automorphisms of $\mathbf{C}^{n}$. Invent. Math., 110(2):371-388, 1992.

[And90] Erik Andersén. Volume-preserving automorphisms of $\mathbf{C}^{n}$. Complex Variables Theory Appl., 14(1-4):223235, 1990.

[And00] Erik Andersén. Complete vector fields on $\left(\mathbf{C}^{*}\right)^{n}$. Proc. Amer. Math. Soc., 128(4):1079-1085, 2000.

[AZ13] I. Arzhantsev and M. Zaidenberg. Acyclic curves and group actions on affine toric surfaces. In Affine Algebraic Geometry, pages 1-41. World Scientific Publ., 2013.

[AZK12] I. V. Arzhantsev, M. G. Zaŭdenberg, and K. G. Kuyumzhiyan. Flag varieties, toric varieties, and suspensions: three examples of infinite transitivity. Mat. Sb., 203(7):3-30, 2012.

[Bru04] Marco Brunella. Complete polynomial vector fields on the complex plane. Topology, 43(2):433-445, 2004.

[CLS11] David A. Cox, John B. Little, and Henry K. Schenck. Toric varieties, volume 124 of Graduate Studies in Mathematics. American Mathematical Society, Providence, RI, 2011.

[Dem70] Michel Demazure. Sous-groupes algébriques de rang maximum du groupe de Cremona. Ann. Sci. École Norm. Sup. (4), 3:507-588, 1970. 
[FKZ08] Hubert Flenner, Shulim Kaliman, and Mikhail Zaidenberg. Uniqueness of $\mathbb{C}^{*}$ - and $\mathbb{C}_{+}$-actions on Gizatullin surfaces. Transform. Groups, 13(2):305-354, 2008.

[For11] Franc Forstnerič. Stein manifolds and holomorphic mappings, volume 56 of Ergebnisse der Mathematik und ihrer Grenzgebiete. 3. Folge. A Series of Modern Surveys in Mathematics. Springer, Heidelberg, 2011.

[For13] Franc Forstnerič. Oka manifolds: from Oka to Stein and back. Ann. Fac. Sci. Toulouse Math. (6), 22(4):747-809, 2013. With an appendix by Finnur Lárusson.

[FR93] Franc Forstnerič and Jean-Pierre Rosay. Approximation of biholomorphic mappings by automorphisms of $\mathbf{C}^{n}$. Invent. Math., 112(2):323-349, 1993.

[Ful93] William Fulton. Introduction to toric varieties, volume 131 of Annals of Mathematics Studies. Princeton University Press, Princeton, NJ, 1993. The William H. Roever Lectures in Geometry.

[Har77] Robin Hartshorne. Algebraic geometry. Graduate Texts in Mathematics, No. 52. Springer-Verlag, New York, 1977.

[KK08] Shulim Kaliman and Frank Kutzschebauch. Criteria for the density property of complex manifolds. Invent. Math., 172(1):71-87, 2008.

[KK11] Shulim Kaliman and Frank Kutzschebauch. On the present state of the Andersén-Lempert theory. In Affine algebraic geometry, volume 54 of CRM Proc. Lecture Notes, pages 85-122. Amer. Math. Soc., Providence, RI, 2011.

[KK12] Shulim Kaliman and Frank Kutzschebauch. On algebraic volume density property, 2012. arXiv:1201.4769 [math.AG].

[Kut14] Frank Kutzschebauch. Flexibility properties in complex analysis and affine algebraic geometry. In $A u$ tomorphisms in Birational and Affine Geometry, pages 387-405. Springer, 2014.

[Lár11] Finnur Lárusson. Smooth toric varieties are Oka, 2011. arXiv:1107.3604 [math.AG].

[Oda88] Tadao Oda. Convex bodies and algebraic geometry. An introduction to the theory of toric varieties, volume 15 of Ergebnisse der Mathematik und ihrer Grenzgebiete (3). Springer-Verlag, Berlin, 1988.

[Ros99] Jean-Pierre Rosay. Automorphisms of $\mathbf{C}^{n}$, a survey of Andersén-Lempert theory and applications. In Complex geometric analysis in Pohang (1997), volume 222 of Contemp. Math., pages 131-145. Amer. Math. Soc., Providence, RI, 1999.

[RR88] Jean-Pierre Rosay and Walter Rudin. Holomorphic maps from $\mathbf{C}^{n}$ to $\mathbf{C}^{n}$. Trans. Amer. Math. Soc., 310(1):47-86, 1988

[Suz77] Masakazu Suzuki. Sur les opérations holomorphes du groupe additif complexe sur l'espace de deux variables complexes. Ann. Sci. École Norm. Sup. (4), 10(4):517-546, 1977.

[Var01] Dror Varolin. The density property for complex manifolds and geometric structures. J. Geom. Anal., 11(1):135-160, 2001.

Mathematisches Institut, Universität Bern, Sidlerstrasse 5, CH-3012 Bern, Switzerland.

E-mail address: frank.kutzschebauch@math.unibe.ch

Mathematisches Institut, Universität Bern, Sidlerstrasse 5, CH-3012 Bern, Switzerland.

E-mail address: matthias.leuenberger@math.unibe.ch

Instituto de Matemática y Física, Universidad de Talca, Casilla 721, Talca, Chile.

E-mail address: aliendo@inst-mat.utalca.cl 